\title{
Digital Papillary Adenocarcinoma: Case of a Rare Malignant Cutaneous Tumor of the Eccrine Sweat Gland
}

\author{
Austin J. Jabbour ${ }^{a}$ Ibrahim A. Tangoren ${ }^{b}$ Andrew B. Kanik ${ }^{c}$ \\ aThe University of Queensland-Ochsner Clinical School, New Orleans, LA, USA; bPrivate \\ Practice, I.A. Tangoren, PLLC, Dermatology and Dermatologic Surgery, Syracuse, NY, USA; \\ 'Private Practice, CBLPath, Rye Brook, NY, USA
}

\section{Keywords}

Digital papillary adenocarcinoma $\cdot$ Cutaneous adnexal tumors $\cdot$ Skin cancer

\begin{abstract}
Digital papillary adenocarcinoma (DPA) is a rare cutaneous tumor originating from the eccrine sweat glands. These lesions occur almost exclusively on the digits of the hands and feet, where there is a high concentration of eccrine glands. The diagnosis is made histologically, and the course of the malignancy tends to be very aggressive with high rates of recurrence and early metastasis at the time of diagnosis. Due to the low incidence of these lesions, there have been minimal objective data from clinical studies to recommend specific treatment strategies. Wide local excision versus digital amputation proximal to the lesion has been debated for primary treatment, while there are no data to support routine implementation of adjuvant chemotherapy or radiation, despite its metastatic nature. This article presents a case of long-standing, previously undiagnosed DPA. The lesion appeared more inconspicuous on gross examination than other reports in the literature, and diagnosis was made with punch biopsy and confirmed postsurgically. To date, the patient has not had recurrence, although she is being monitored for potential metastatic deposits in her lungs. Clinical dermatologists should be aware of the high mortality burden this lesion may inflict if left undiagnosed or mistreated.
\end{abstract}

\section{Introduction}

Digital papillary adenocarcinoma (DPA) is a rare tumor originating from the eccrine sweat glands that often presents as a solitary, often painless mass. These lesions tend to appear almost exclusively on the palmar surface of the hands or the plantar aspect of the feet, 
most commonly along the digits [1]. Demographic data available suggest an overlying Caucasian male predominance in their middle to later years of adulthood [2-4]. The typical appearance of the lesion under a microscope consists of multinodular, solid, and cystic tumor components with papillary projections into the cystic spaces. Notably, characteristic patterns of fused back-to-back glands can be seen in solid portions of the tumor [2].

DPA presents challenges to clinical decision-making as these tumors have a high local recurrence rate, up to $50 \%$, and the ability to metastasize throughout the body, most commonly seen in the lymph nodes and lungs [2,3]. Wide local excision (WLE) with clear resection of margins versus proximal amputation is recommended as a first-line surgical approach, and there are no current data to support the regular use of adjuvant chemotherapy or radiation [5]. Regular surveillance with a multidisciplinary team, including dermatology and oncology, is recommended thereafter. Here, we present a case of long-standing DPA, including the presentation, histopathology, diagnostic workup, therapeutic strategies, and complications faced, followed by a discussion of the current treatment strategies and a review of the literature.

\section{Case Report}

A 46-year-old Caucasian female patient with a dermatological history of actinic keratoses and rosacea and a positive family history of melanoma in her paternal uncle presented to a dermatologist for evaluation of a skin lesion located on the dorsal aspect of the left third finger overlying the proximal interphalangeal joint that had been present for the last 11 years (Fig. 1a). The patient had treated this lesion intermittently with over-the-counter wart medication, without any significant improvement. Over several months leading up to presentation, the lesion had become increasingly irritated, erythematous, and pruritic. A shave biopsy was performed at the time of presentation, with dermatopathology revealing hyperkeratosis, papillomatosis, and acanthosis with the top of a dermal cystic lesion lined by the dysplastic epithelium forming papillary fronds without stroma plus focally cystic comedo necrosis, consistent with an aggressive DPA (Fig. 2a, b). The lesion extended to the deep tissue edge of the biopsy specimen. Re-examination of the patient following diagnosis failed to identify lymphadenopathy or hepatosplenomegaly.

The patient was referred to hematology/oncology services for further evaluation of the malignancy. Physical examination revealed no evidence of metastatic disease, but the length of time that the lesion had been present provided some cause for concern. WLE versus digital amputation was discussed at length, with the decision to treat with WLE plus skin grafting for closure and left axillary lymph node dissection by a combination surgical oncology and orthopedic surgery services (Fig. 1b). Sentinel lymph node biopsy (SLNB) was negative at that time. Assessment for potential metastatic disease revealed two-subcentimeter lung lesions on computed tomography scan of the chest, which were monitored with serial computed tomography imaging every 3 months for 1 year without significant changes. The patient was also seen by her dermatologist every 3 months for 1 year following initial treatment and has been returning for annual skin checks since.

Six months following the lymph node evaluation, the patient presented to her surgeon complaining of some difficulty using her left hand. Physical examination revealed mild to moderate lymphedema in the left upper extremity. She was referred to physical therapy for active and passive range of motion and supportive care, including compressive bandages and elevation. On re-evaluation 4 months later, the patient's functional status had significantly improved; she was using her hand without difficulty and minimizing edema with compression. To date of this report, the patient has not suffered any other complications from treatment nor has metastatic disease become evident in follow-up examinations.

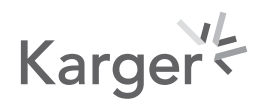


Fig. 1. DPA. a Long-standing skin lesion overlying the PIP joint of the third digit on the left hand, which had become increasingly erythematous and pruritic over several months. b Visual inspection 1 month following WLE of the lesion and skin grafting of the defect. DPA, digital papillary adenocarcinoma; WLE, wide local excision; PIP, proximal interphalangeal.
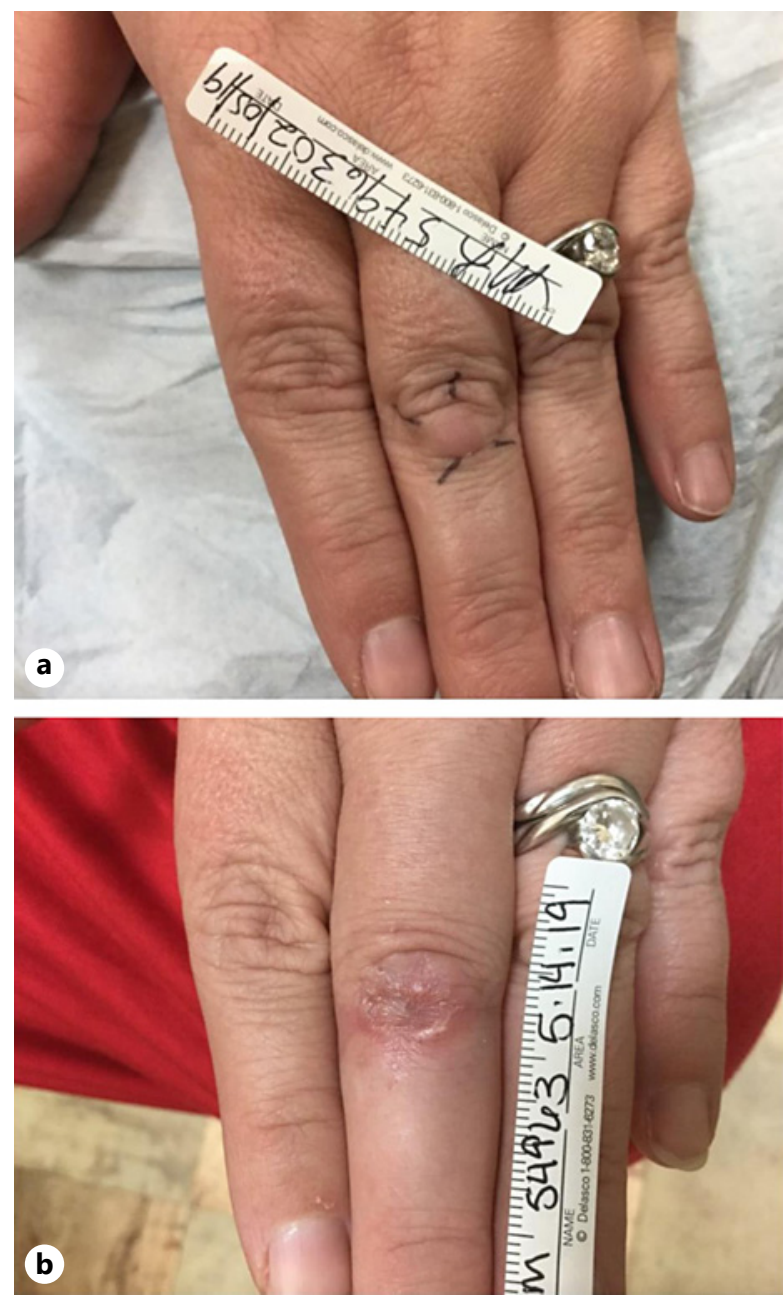

\section{Discussion}

DPA was initially described in 1979 by E.B. Helwig in a series of patients with similar clinical and histopathological findings [4]. These lesions represented a separate histological identity compared to the similar presentation of the eccrine acrospiroma. Further classification of benign versus malignant digital papillary tumors based on histological evidence has been attempted, with the former being named an aggressive digital papillary adenoma and the latter an aggressive digital papillary adenocarcinoma [3]. As with other carcinomas, malignant histology includes poor glandular differentiation, the presence of necrosis, cellular atypia, and pleomorphism, as well as the ability to invade nearby structures. However, making this distinction based on histology alone has been contentious as there has been no reliable correlation between histologic findings and probability of recurrent disease or metastasis [2]. The initial designation as "aggressive" was also removed in 2012 due to the implicit malignant nature the name adenocarcinoma instills [6].

The difficulty in classifying lesions as benign or malignant has also posed problems for clinicians making treatment decisions that will be most beneficial to the patient. The inability to predict outcomes based on clinical and histologic features coupled with high recurrence rates and metastatic disease potential decades after initial treatment would make any physician wary of being too conservative with treatment [5]. Based on a recent population-based 
Fig. 2. DPA. Shave biopsy specimen stained with H\&E. a $\times 10$ magnification showing cystic comedo necrosis (yellow arrow) surrounded by the dysplastic epithelium (blue arrow). $\mathbf{b} \times 40$ magnification showing the irregular epithelium forming papillary fronds without stroma (red arrow). DPA, digital papillary adenocarcinoma.
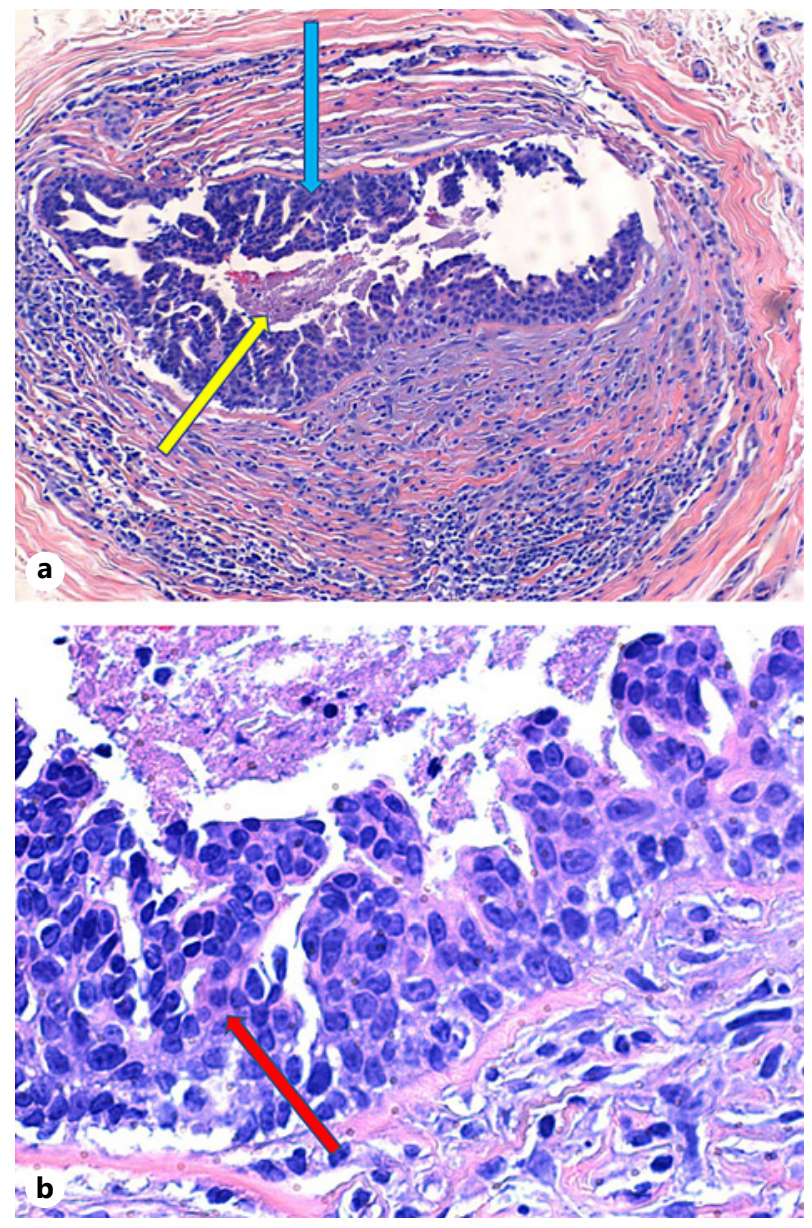

study of digital papillary adenocarcinoma, regional disease spread occurred in $22.3 \%$ of afflicted individuals, while disease-specific mortality was seen in $2.1 \%$ of patients [7]. However, a retrospective case series of 19 cases of DPA concluded that there was no significant difference in recurrence rates between conservative and aggressive surgical treatment strategies [8].

When taken in the context of this case, the length of time the lesion was present prior to diagnosis suggested that metastatic disease was more likely, warranting SLNB and wholebody metastatic screening. However, there are still no guidelines to aid in these decisions, and it is left up to the provider team on a case-by-case scenario. A case series of upper extremity DPA published by Bartelstein et al. [9] in 2020 demonstrated a significant association between positive SLNB and systemic recurrence, making the argument that SLNB could be routinely employed for staging of disease at the time of diagnosis. There have been multiple reports on metastatic disease discovered up to 20 years after initial presentation, necessitating longterm follow-up with clinical examinations and combined imaging modalities [6, 10]. Unfortunately, no criteria for follow-up end points have been established. For this patient, it was deemed appropriate to follow up annually with both her dermatologist and oncologist indefinitely to screen for local recurrence and metastatic spread.

Future studies should be directed at evidence-based trials with objective outcome measures to determine the most effective form of primary treatment and whether systemic or localized adjuvant treatment would decrease the risk of recurrent or metastatic disease. The role of chemotherapy to treat these malignancies has been poorly documented and 
remains unclear. One case of DPA with pulmonary metastases was treated with a combination chemotherapy regimen consisting of cisplatin and gemcitabine without any significant improvement in the metastatic lesions [11]. Two other cases were treated with a combination of interferon, doxorubicin, and platinum-based agents, to which a modest benefit was seen [12]. As the incidence of this disease is extremely low, estimated to be 0.08 cases per 1 million person-years [7], it is unlikely that pharmaceutical research and development would reveal new drug targets specific to DPA, so repurposing of existing therapies would be better suited for trials.

\section{Statement of Ethics}

All procedures adopted in this study were conducted ethically in accordance with the World Medical Association Declaration of Helsinki. Written informed consent was obtained from the patient for publication of this case report and any accompanying images. The study is exempt from ethical committee approval as the patient was treated with the standard of care using evidence-based guidelines.

\section{Conflict of Interest Statement}

The authors have no conflicts of interest to declare.

\section{Funding Sources}

The authors did not receive any funding to conduct this research.

\section{Author Contributions}

A.J. Jabbour saw the patient, compiled medical records, and was the major contributor in writing the manuscript. I.A. Tangoren was the primary dermatologist involved in the diagnosis and initial care of this patient. A.B. Kanik was the dermatopathologist who made the histologic diagnosis and provided all images related to the case.

\section{Data Availability Statement}

No datasets were generated or analyzed during the current study.

\section{References}

1 Carter K, Yao JJ, Melton SD, Lopez J, Huerta S. Digital papillary adenocarcinoma: presentation, natural history and management. Rare Tumors. 2015;7(2):5867-95.

2 Duke WH, Sherrod TT, Lupton GP. Aggressive digital papillary adenocarcinoma (aggressive digital papillary adenoma and adenocarcinoma revisited). Am J Surg Pathol. 2000;24(6):775-84.

3 Kao GF, Helwig EB, Graham JH. Aggressive digital papillary adenoma and adenocarcinoma. A clinicopathological study of 57 patients, with histochemical, immunopathological, and ultrastructural observations. J Cutan Pathol. 1987;14(3):129-46.

4 Helwig EB. Eccrine acrospiroma. J Cutan Pathol. 1984;11(5):415-20. 
5 Vale C, Carvalho A, Roseiro T, Antunes J, Tellechea Ó, Oliveira V. Digital papillary adenocarcinoma, a rare malignant tumor. Case Rep Orthop Res. 2019;2(1-3):89-93.

6 Suchak R, Wang WL, Prieto VG, Ivan D, Lazar AJ, Brenn T, et al. Cutaneous digital papillary adenocarcinoma: a clinicopathologic study of 31 cases of a rare neoplasm with new observations. Am J Surg Pathol. 2012;36(12): 1883-91.

7 Rismiller K, Knackstedt TJ. Aggressive digital papillary adenocarcinoma: population-based analysis of incidence, demographics, treatment, and outcomes. Dermatol Surg. 2018;44(7):911-7.

8 Weingertner N, Gressel A, Battistella M, Cribier B. Aggressive digital papillary adenocarcinoma: a clinicopathological study of 19 cases. J Am Acad Dermatol. 2017;77(3):549-e1.

9 Bartelstein MK, Schwarzkopf E, Busam KJ, Brady MS, Athanasian EA. Sentinel lymph node biopsy predicts systemic recurrence in digital papillary adenocarcinoma. J Surg Oncol. 2020;122(7):1323-7.

10 Jones JA, Patel VB, Goldsmith B, Teitelbaum U, Plastaras JP. Diffusely metastatic digital papillary adenocarcinoma 11 years after initial presentation treated with palliative chemotherapy and radiotherapy. J Clin Oncol. 2013;31(22):e386-9.

11 Nishimoto J, Amoh Y, Niiyama S, Takasu H, Katsuoka K. Aggressive digital papillary adenocarcinoma on the palm with pulmonary metastases. J Dermatol. 2008;35(7):468-70.

12 Bakotic B, Antonescu CR. Aggressive digital papillary adenocarcinoma of the foot: the clinicopathologic features of two cases. J Foot Ankle Surg. 2000;39(6):402-5. 